\title{
XY Genotype
}

National Cancer Institute

\section{Source}

National Cancer Institute. XY Genotype. NCI Thesaurus. Code C45977.

The normal genotype of a male human. 